\title{
NOUVELLE
}

\section{L'histoire controversée du facteur trypanolytique humain}

Etienne Pays, Benoit Vanhollebeke
Laboratoire de Parasitologie Moléculaire, IBMM,

Université Libre de Bruxelles,

12 , rue des Professeurs Jeener et Brachet, B6041 Gosselies, Belgique.

epays@ulb.ac.be
$>$ Les trypanosomes africains (prototype Trypanosoma brucei) sont des protozoaires flagellés capables d'infecter de nombreux mammifères. Chez l'homme, ces parasites provoquent la maladie du sommeil, fatale si elle n'est pas traitée précocement. Dès 1900, Laveran et Mesnil notent qu'en fait l'homme est immun contre $T$. $b$. brucei, et que seules des formes variantes dérivées de cet organisme, maintenant appelées $T$. $b$. rhodesiense et $T$. b. gambiense, sont capables d'échapper à notre défense innée [1].

\section{Trente ans d'histoire controversée} du facteur trypanolytique

L'identification du facteur sérique humain capable de tuer T. b. brucei a été l'objet d'une quête laborieuse et mouvementée. Le travail pionnier de Mary Rifkin établit que ce facteur est associé à des particules d'HDL (high density lipo- proteins) [2], puis différents groupes concluent que curieusement, les particules d'HDL trypanolytiques se fixent à un récepteur de surface du parasite pour être activement intériorisées par la voie endocytaire [3]. L'acidification inhérente au processus de digestion est requise pour que le facteur lyse le parasite, et le lysosome, destination finale du trafic intracellulaire, apparaît comme étant le compartiment cible de l'activité lytique [3]. Les tentatives de purification du facteur lytique concluent que ce dernier est une protéine spécifiquement humaine, très similaire à l'haptoglobine, l'haptoglobin-related protein ou $\mathrm{Hpr}$, et le mécanisme de trypanolyse invoqué est une peroxidation de la membrane du lysosome due à I'hémoglobine ( $\mathrm{Hb})$ associée à l'Hpr [4]. Outre la fraction associée à des HDL, un autre complexe sérique trypanolytique

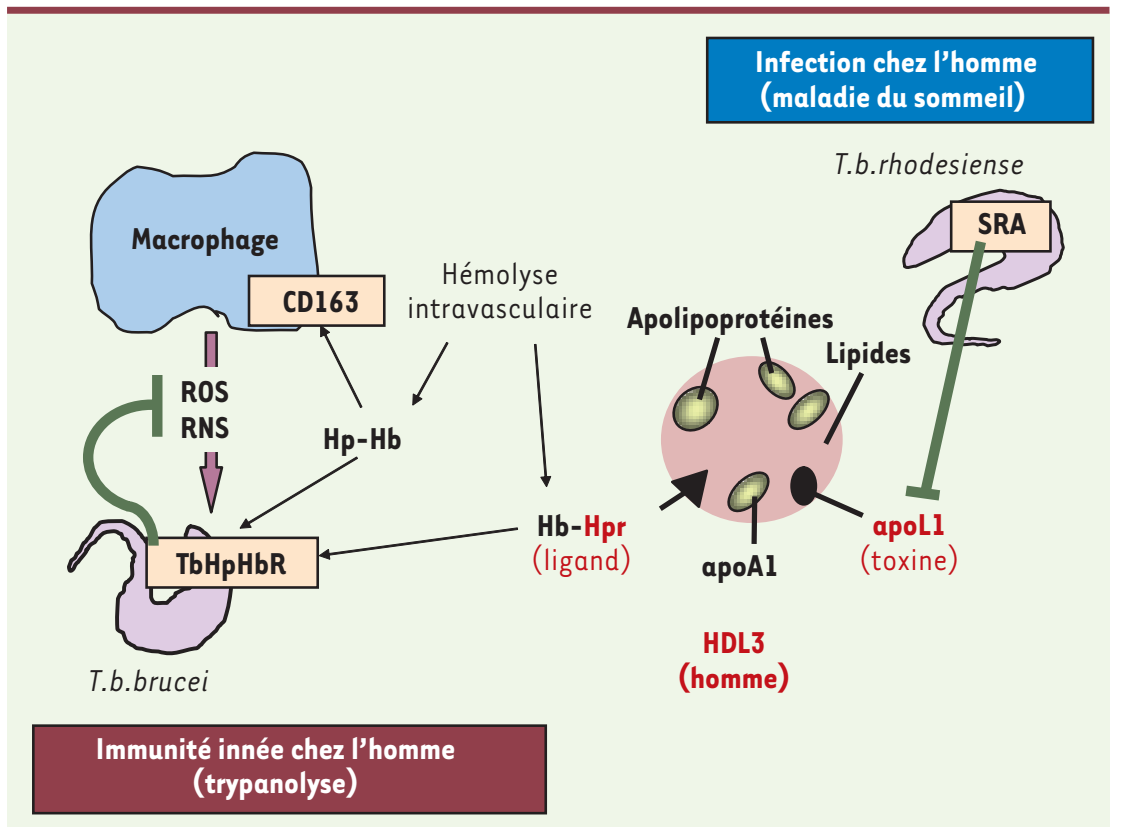

contenant l'Hpr est également identifié, et les deux fractions sont respectivement baptisées TLF (trypanosome lytic factor)1 et TLF-2 [5]. Des conclusions complètement différentes sont obtenues à la suite de l'identification de la protéine permettant à $T$. $b$. rhodesiense de résister au facteur trypanolytique [6]. Cette protéine, appelée SRA pour serum-resistance associated protein s'avère interagir fortement avec l'apolipoprotéine Ll (apoll), qui, comme l'Hpr, est une protéine spécifiquement humaine associée aux HDL [7]. L'interaction entre SRA et apoLl, qui a lieu dans le lysosome du parasite, conditionne la résistance de ce dernier au sérum humain. De plus, l'étude détaillée de l'apoLl établit que cette protéine est le facteur trypanolytique tant recherché [7]. L'apoLl tue le trypanosome par gonflement osmotique du lysosome résultant de l'activité formatrice de pores anioniques par cette protéine [8].

Hpr et Apolipoprotéine L1, une association mortelle pour le parasite Dans un numéro récent du journal Science, nous publions la découverte du récepteur de surface cellulaire qui permet la capture des HDL trypanolytiques

Figure 1. Le facteur trypanolytique humain. Le rôle de ligand joué par l'Hpr dans les particules trypanolytiques explique l'effet accélérateur de cette protéine dans le processus de trypanolyse. Les particules d'HDL trypanolytiques sont constituées de deux protéines humaines à fonctions distinctes: I'Hpr, qui permet l'entrée efficace et rapide des particules dans le parasite, et l'apoLl, la toxine qui tue le trypanosome 
par T.b. brucei [9]. Ce travail révèle que ce récepteur reconnaît spécifiquement le complexe Hpr-Hb. Étant donné que les particules d'HDL porteuses d'Hpr-Hb contiennent aussi l'apoLl [10], la présence de ce récepteur entraîne la fixation membranaire, puis l'intériorisation des particules d'HDL trypanolytiques dans le parasite. Ces observations permettent de réconcilier les conclusions apparemment contradictoires présentées jusqu'ici. En effet, le rôle de ligand joué par l'Hpr dans les particules trypanolytiques explique l'effet accélérateur de cette protéine dans le processus de trypanolyse, erronément interprété initialement comme étant dû à une activité lytique intrinsèque. L'activité lytique semble exclusivement due à l'apoLl [11], mais en l'absence d'Hpr, cette protéine ne pénètre que très difficilement dans le trypanosome. Les particules d'HDL trypanolytiques sont donc constituées de deux protéines humaines à fonctions distinctes: I'Hpr est la clé qui permet l'entrée efficace et rapide des particules dans le parasite, et l'apoLl est la toxine qui tue le trypanosome (Figure 1). On peut considérer l'invention de ces particules originales comme un nouveau mécanisme d'immunité innée contre les trypanosomes, développé chez l'homme et certains grands singes africains (comme le gorille), peut-être en raison du fait que ces parasites sont endémiques sur le lieu d'apparition évolutive de l'homme.
Le récepteur $\mathrm{Hpr}-\mathrm{Hb}$, un rôle ambigu... Le récepteur $\mathrm{Hpr}-\mathrm{Hb}$ de T.b. brucei n'a évidemment pas pour fonction primaire d'assurer l'auto-destruction du parasite. Dans le même travail [9], nous montrons qu'en fait ce récepteur reconnaît aussi le complexe $\mathrm{Hp}$ (haptoglobine)-Hb, et que l'intériorisation de ce complexe permet de fournir de l'hème au parasite. Cette molécule est apparemment incorporée dans des hémoprotéines qui contribuent à rendre le trypanosome moins sensible à la réponse oxydante de l'hôte. Étant donné que les macrophages de l'hôte possèdent aussi un récepteur (CD163) pour le même complexe, le trypanosome a dû élaborer un récepteur possédant une affinité comparable pour ce ligand. Cependant, le récepteur parasitaire se distingue du récepteur de l'hôte par sa capacité à reconnaître aussi le complexe $\mathrm{Hpr}-\mathrm{Hb}$.

$\varepsilon n$ conclusion, avant l'apparition de l'homme, le récepteur $\mathrm{Hp}-\mathrm{Hb}$ était bénéfique pour le parasite car il facilitait sa prolifération dans l'hôte en lui permettant de mieux résister aux macrophages. Chez l'homme, la présence simultanée d'Hpr et d'apoll sur une fraction des HDL a détourné la fonction initiale du récepteur en organisant la capture de particules trypanolytiques par le parasite. La réponse du trypanosome n'a pas tardé, puisqu'au moins deux clones ont élaboré un mécanisme de résistance, ce qui leur permet de croître chez l'homme et de provoquer la maladie du sommeil. $\diamond$
The controversial story

of the human trypanolytic factor

\section{RÉFÉRENCES}

1. Laveran A, Mesnil F. Trypanosomes et trypanosomiases. Paris : Masson, 1912 : $1000 \mathrm{p}$.

2. Rifkin MR. Identification of the trypanocidal factor in normal human serum : high density lipoprotein. Proc Natl Acad. Sci USA $1978 ; 75$ : 3450-4.

3. Hager KM, Pierce MA, Moore DR, et al. Endocytosis of a cytotoxic human high density lipoprotein results in disruption of acidic intracellular vesicles and subsequent killing of African trypanosomes. J Cell Biol $1994 ; 126: 155-67$.

4. Smith AB, Esko JD, Hajduk SL. Killing of trypanosomes by the human haptoglobin-related protein. Science 1995 ; 268 : 284-6.

5. Raper J, Fung R, Ghiso J, et al. Characterization of a novel trypanosome lytic factor from human serum. Infect Immun 1999; 67 : 1910-6.

6. Xong HV, Vanhamme L, Chamekh M, et al. A VSG expression site-associated gene confers resistance to human serum in Trypanosoma rhodesiense. Cell 1998 ; $95: 839-46$.

7. Vanhamme L, Paturiaux-Hanocq F, Poelvoorde P, et al. Apolipoprotein L-I is the trypanosome lytic factor of human serum. Nature $2003 ; 422: 83-7$.

8. Pérez-Morga D, Vanhollebeke B, Paturiaux-Hanocq F, et al. Apolipoprotein L-I promotes trypanosome lysis by forming pores in lysosomal membranes. Science $2005 ; 309: 469-72$.

9. Vanhollebeke B, Demuylder G, Nielsen, MJ, et al. A haptoglobin-hemoglobin receptor conveys innate immunity to Trypanosoma brucei in humans. Science $2008 ; 320: 677-81$.

10. Shiflett AM, Bishop JR, Pahwa A, Hajduk SL. Human high density lipoproteins are platforms for the assembly of multi-component innate immune complexes. J Biol Chem 2005 ; 280 : 32578-85.

11. Vanhollebeke B, Nielsen MJ, Watanabe $Y$, et al. Distinct roles of haptoglobin-related protein and apolipoprotein L-I in trypanolysis by human serum. Proc Natl Acad Sci USA 2007 ; 104 : 4118-23.

12. Baral TN, Magez $S$, Stijlemans B, et al. Traitement expérimental de la trypanosomiase africaine par le facteur trypanolytique humain combiné à un nanocorps. Med Sci (Paris) 2006 ; 22 : 914-6.

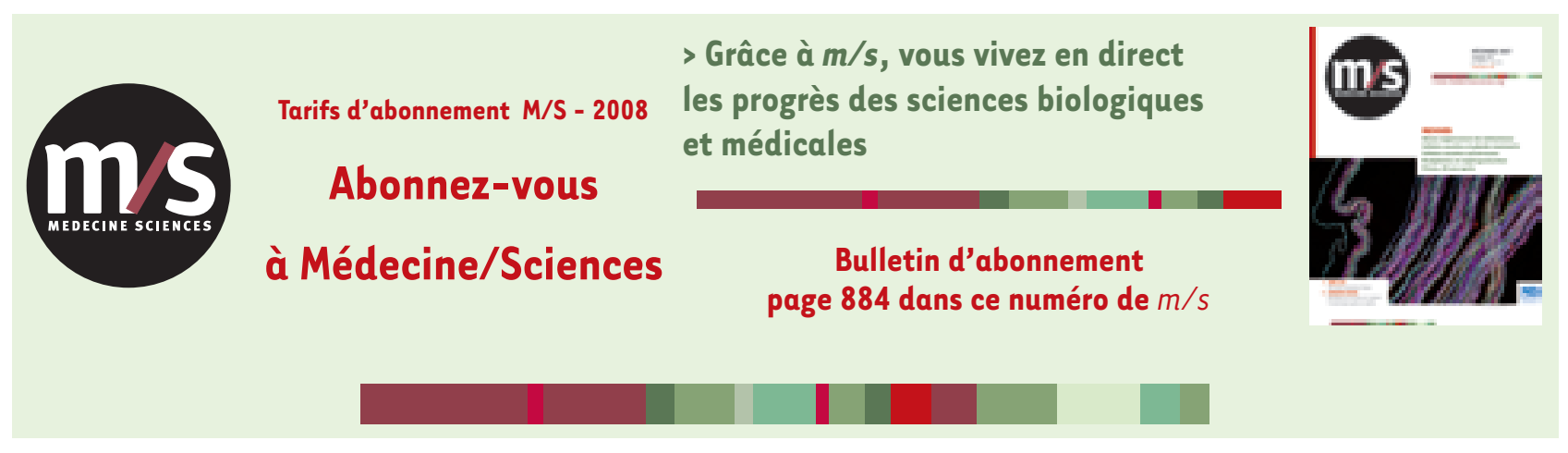

